Eur. J. Math. Appl. (2021)1:4

URL: http://ejma.euap.org

(C) 2021 European Journal of Mathematics and Applications

\title{
THE NEW RESULTS IN $n$-ABELIAN CATEGORY
}

\author{
SAMIRA HASHEMI, FEYSAL HASSANI, AND RASUL RASULI*
}

\begin{abstract}
In this paper, we study the homological theory in $n$-abelian categories. First, we prove some useful properties of $n$-pushout diagram and $n$-pullback diagram. Finally, we recall the definition of theory in $n$-abelian categories.
\end{abstract}

\section{INTRODUCTION}

Category theory formalizes mathematical structures and its concepts in terms of a labeled directed graph called a category, whose nodes are called objects, and their edges called arrows (or morphisms). This category has two basic properties: the ability to compose the arrows associatively and the existence of an identity arrow for each object. The language of category theory has been employed to formalize concepts of other high-level abstractions such as sets, rings, and groups. Several terms were utilized in category theory, including the "morphism" that is used differently from their usage in the rest of mathematics. In category theory, morphisms obey specific conditions of theory. Samuel Eilenberg and Saunders Mac Lane introduced the concepts of categories, functors, and natural transformations in 1942-45 in their study of algebraic topology, to understand the processes that preserve the mathematical structure. Category theory has practical applications in programming language theory, for example, the usage of monads in functional programming. It may also be used as an axiomatic foundation for mathematics, as an alternative to set theory and other proposed foundations. In mathematics, an abelian category is a category in which morphisms and objects can be added and in which kernels and cokernels exist and have desirable properties. The motivating prototype example of an abelian category is the category of abelian groups, Ab. The theory originated to unify several cohomology theories by Alexander Grothendieck and independently in the slightly earlier work of David Buchsbaum. Abelian categories are very stable categories. For example, they are regular and satisfy the snake lemma. The class of Abelian categories is closed under several categorical constructions, for instance, the category of chain complexes of an Abelian category, or the category of functors from a small category to an Abelian category are Abelian as well. These stability properties make them inevitable in homological algebra and beyond. This theory has significant applications in algebraic geometry, cohomology, and pure category

Department of Mathematics, Payame Noor University (PNU), P.O. Box, 19395-4697 Tehran, IRAN

*Corresponding AUTHOR

E-mail addresses: s.hashemi300@gmail.com, hassani@pnu.ac.ir, rasulirasul@yahoo.com.

Key words and phrases. $n$-ker and $n$-coker; $n$-exact sequence; $n$-abelian category; $n$-pushout diagram; $n$ pullback diagram.

Received 08/08/2021. 
theory. The Abelian categories are named after Niels Henrik Abel. An exact sequence is a concept in mathematics, especially in group theory, ring, and module theory, homological algebra, as well as in differential geometry. An exact sequence is a sequence, either finite or infinite, of objects and morphisms between them such that the image of one morphism equals the kernel of the next. Homological algebra is the branch of mathematics that studies homology in a general algebraic setting. It is a relatively young discipline, whose origins can be traced to investigations in combinatorial topology (a precursor to algebraic topology) and abstract algebra (theory of modules and syzygies) at the end of the 19th century, chiefly by Henri Poincar'e and David Hilbert. The development of homological algebra has closely intertwined with the emergence of category theory. By and large, homological algebra is the study of homological functors and the intricate algebraic structures that they entail. One quite useful and ubiquitous concept in mathematics is that of chain complexes, which can be studied both through their homology and cohomology. Homological algebra affords the means to extract information contained in these complexes and present it in the form of homological invariants of rings, modules, topological spaces, and other 'tangible' mathematical objects. A powerful tool for doing this is provided by spectral sequences. From its very origins, homological algebra has played an enormous role in algebraic topology. Its sphere of influence has gradually expanded and presently includes commutative algebra, algebraic geometry, algebraic number theory, representation theory, mathematical physics, operator algebras, complex analysis, and the theory of partial differential equations. K-theory is an independent discipline that draws upon methods of homological algebra, as does the noncommutative geometry of Alain Connes.

Abelian categories were introduced by Grothendieck in [7] to axiomatize the properties of the category of modules over a ring and of the category of sheaves over a scheme. It is often the case that interesting additive categories are not abelian but still have good homological properties with respect to a restricted class of short exact sequences.

In this paper in section 2 we show to prove the important theorems of $n$-pushout diagram, $n$ pullback diagram and in section 3 we show that any small $n$-abelian category can be embedded in an abelian category in such a way that the embedding functor is $n$-exact and reflects $n$ exact sequences. We introduce $n$-abelian categories and establish their basic properties; we give a characterization of semisimple categories in terms of $n$-abelian categories.

\section{Preliminaries}

All rings $R$ in this paper are assumed to have an identity element 1 (or unit) (where $r 1=$ $r=1 r$ for all $r \in R$ ). We do not insist that $1 \neq 0$; however, should $1=0$, then $R$ is the zero ring having only one element.

In this section, we recall some of the fundamental concepts and definitions, which are necessary for this paper. For details, we refer to $[2,3,5,11,12]$.

Definition 2.1. A category $\mathcal{C}$ is abelian if

(1) $\mathcal{C}$ has a zero object.

(2) For every pair of objects there is a product and a sum.

(3) $\mathcal{C}$ Every map has a kernel and cokernel. 
(4) $\mathcal{C}$ Every monomorphism is a kernel of a map.

(5) $\mathcal{C}$ Every epimorphism is a cokernel of a map.

Definition 2.2. Let $\mathcal{C}$ be an additive category and $f: A \longrightarrow B$ a morphism in $\mathcal{C}$. A weak cokernel of $f$ is a morphism $g: B \longrightarrow C$ such that for all $C^{\prime} \in \mathcal{C}$ the sequence of abelian groups

$$
\mathcal{C}\left(C, C^{\prime}\right) \stackrel{\hat{g}}{\longrightarrow} \mathcal{C}\left(B, C^{\prime}\right) \stackrel{\hat{f}}{\longrightarrow} \mathcal{C}\left(A, C^{\prime}\right)
$$

is exact. Equivalently, $g$ is a weak cokernel of $\mathrm{f}$ if $f g=0$ and for each morphism $h: B \longrightarrow C^{\prime}$ such that $f h=0$ there exists a (not necessarily unique) morphism $p: C \longrightarrow C^{\prime}$ such that $h=g p$. These properties are subsumed in the following commutative diagram:

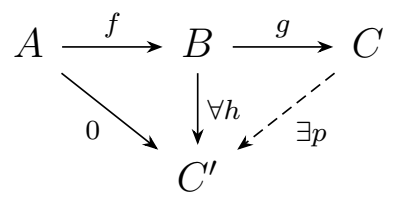

Clearly, a weak cokernel $g$ of $f$ is a cokernel of $f$ if and only if $g$ is an epimorphism. The concept of weak kernel is

defined dually.

Definition 2.3. Let $\mathcal{C}$ be an additive category and $d^{0}: X^{0} \longrightarrow X^{1}$ a morphism in $\mathcal{C}$. An $n$-coker of $d^{0}$ is a sequence

$$
\left(d^{1}, \ldots, d^{n}\right): X^{1} \stackrel{d^{1}}{\longrightarrow} X^{2} \stackrel{d^{2}}{\longrightarrow} \ldots \stackrel{d^{n}}{\longrightarrow} X^{n+1}
$$

such that, , for all $Y \in \mathcal{C}$ the induced sequence of abelian groups

$$
0 \longrightarrow \mathcal{C}\left(X^{n+1}, Y\right) \stackrel{\hat{d}^{n}}{\longrightarrow} \mathcal{C}\left(X^{n}, Y\right) \stackrel{\hat{d}^{n-1}}{\longrightarrow} \ldots \stackrel{\hat{d}^{1}}{\longrightarrow} \mathcal{C}\left(X^{1}, Y\right) \stackrel{\hat{d}^{0}}{\longrightarrow} \mathcal{C}\left(X^{0}, Y\right)
$$

is exact. Equivalently, the sequence $\left(d^{1}, \ldots, d^{n}\right)$ is an $n$-coker of $d^{0}$ if, for all $1 \leq k \leq n-1$ the morphism $d^{k}$ is a weak cokernel of $d^{k-1}$, and $d^{n}$ is moreover a cokernel of $d^{n-1}$. In this case, we say the sequence

$$
X^{0} \stackrel{d^{0}}{\longrightarrow} X^{1} \stackrel{d^{1}}{\longrightarrow} X^{2} \stackrel{d^{2}}{\longrightarrow} \ldots \stackrel{d^{n}}{\longrightarrow} X^{n+1}
$$

is right n-exact.

Remark 2.4. When we say $n$-cokernel we always means that $n$ is a positive integer. We note that the notion of 1 -cokernel is the same as cokernel. we can define $n$ - kernel and left $n$-exact sequence dually.

Definition 2.5. Let $\mathcal{C}$ be an additive category. An $n$-exact sequence in $\mathcal{C}$ is a complex

$$
X^{0} \stackrel{d^{0}}{\longrightarrow} X^{1} \stackrel{d^{1}}{\longrightarrow} \ldots \stackrel{d^{n-1}}{\longrightarrow} X^{n} \stackrel{d^{n}}{\longrightarrow} X^{n+1}
$$

in $C h^{n}(\mathcal{C})$ such that $\left(d^{0}, \ldots, d^{n-1}\right)$ is an $n$-ker of $d^{n}$, and $\left(d^{1}, \ldots, d^{n}\right)$ is an $n$-coker of $d^{0}$. The sequence (3.1) is called $n$-exact if it is both right $n$-exact and left $n$-exact.

Definition 2.6. Let $\mathcal{C}$ be an additive category. A pushout diagram of a pair of morphisms

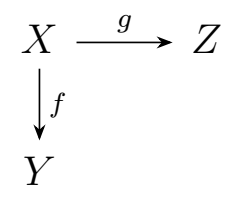


in $\mathcal{C}$ can be identified with a cokernel of the morphism $[-g f]^{\top}: X \longrightarrow Z \oplus Y$. This motivates us to introduce the following concept.

Definition 2.7. An additive category $\mathcal{C}$ is semisimple if every morphism $f: A \longrightarrow B$ in $\mathcal{C}$ factors as $f=p i$, where $p$ is a split epimorphism and $i$ is a split monomorphism.

Lemma 2.8. Let $\mathcal{C}$ be an additive category and $X \in C h^{\geq 0}(\mathcal{C})$ a complex such that for all $k \geq 0$ the morphism $d_{X}^{k+1}$ is a weak cokernel of $d_{X}^{k}$. If $f: X \longrightarrow Y$ and $g: X \longrightarrow Y$ are morphisms in $C h^{\geq 0}(\mathcal{C})$ such that $f^{0}=g^{0}$, then there exists a homotopy $h: f \longrightarrow g$ such that $h^{1}$ is the zero morphism.

Proposition 2.9. Let $\mathcal{C}$ be an additive category and $X$ and $Y$ be com plexes in $C h^{n}(\mathcal{C})$ which are isomorphic in in $H(\mathcal{C})$. Then the following statements hold.

(1) The complex $X$ is an $n$-exact sequence if and only if $Y$ is an n-exact sequence.

(2) Every contractible complex with $n+2$ terms is an $n$-exact sequence.

Proposition 2.10. Let $\mathcal{C}$ be an additive category and $X$ a complex in $C h^{n}(\mathcal{C})$ such that $\left(d^{1}, \ldots, d^{n}\right)$ is an $n$-cokernel of $d^{0}$. Then, $d^{0}$ is a split monomorphism if and only if $X$ is a contractible n-exact sequence.

Proposition 2.11. Let $\mathcal{C}$ be an additive category and $f: X \longrightarrow Y$ a morphism of $n$-exact sequences in $\mathcal{C}$ such that $f^{k}$ and $f^{k+1}$ are isomorphisms for some $k \in\{1, \ldots, n\}$. Then, $f$ induces an isomorphism in $H(\mathcal{C})$.

Proposition 2.12. Let $f$ be a morphism in the quasi-abelian category $\mathcal{C}$. The canonical morphism $\hat{f}:$ Coim $f \longrightarrow$ Imf is monomorphism and epimorphism.

Proof. By duality it suffices to check that the morphism $\bar{f}$ in the diagram

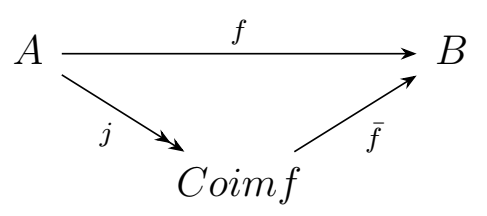

is monomorphism. Let $x: X \longrightarrow$ Coimf be a morphism such that $\bar{f} x=0$. The pull-back $y: Y \longrightarrow A$ of $x$ along $j$ satisfies $f y=0$, so $y$ factors over ker $f$ and hence $j y=0$. But then the map $Y \rightarrow X \longrightarrow$ Coimf is zero as well,so $x=0$.

\section{N-PUSHOUT DIAGRAM AND N-PULLBACK DIAGRAM}

Definition 3.1. Let $n$ be a positive integer. An $n$-abelian category is an additive category $\mathcal{C}$ which satisfies the following axioms;

$\left(A^{0}\right)$ The category $\mathcal{C}$ is idempotent complete.

$\left(A^{1}\right)$ Every morphism in $\mathcal{C}$ has $n$-ker and $n$-coker.

$\left(A^{2}\right)$ For every monomorphism $f^{0}: X^{0} \longrightarrow X^{1}$ in $\mathcal{C}$ and, for every $n$-cokernel $\left(f^{0}, f^{1}, \ldots, f^{n-1}\right)$ of $f^{0}$, the following sequence $n$-exact:

$$
X^{0} \stackrel{f^{0}}{\longrightarrow} X^{1} \stackrel{f^{1}}{\longrightarrow} \ldots \stackrel{f^{n-1}}{\longrightarrow} X^{n} \stackrel{f^{n}}{\longrightarrow} X^{n+1}
$$


$\left(A^{2^{o p}}\right)$ For every epimorphism $g^{n}: X^{n} \longrightarrow X^{n+1}$ in $\mathcal{C}$ and, for every $n$-kernel $\left(g^{0}, g^{1}, \ldots, g^{n-1}\right)$ of $g^{n}$, the following sequence $n$-exact:

$$
X^{0} \stackrel{g^{0}}{\longrightarrow} X^{1} \stackrel{g^{1}}{\longrightarrow} \ldots \stackrel{g^{n-1}}{\longrightarrow} X^{n} \stackrel{g^{n}}{\longrightarrow} X^{n+1}
$$

Let us give some important remarks regarding Definition 3.1.

Remark 3.2. By Proposition 2.9 and Proposition 2.11 we can replace axiom $\left(A^{2}\right)$ by the following weaker version:

$\left(A^{2^{*}}\right)$ For every monomorphism $f^{0}: X^{0} \longrightarrow X^{1}$ in $\mathcal{C}$ there exists an $n$-exact sequence:

$$
X^{0} \stackrel{f^{0}}{\longrightarrow} X^{1} \stackrel{f^{1}}{\longrightarrow} \ldots \stackrel{f^{n-1}}{\longrightarrow} X^{n} \stackrel{f^{n}}{\longrightarrow} X^{n+1}
$$

Naturally, we can weaken axiom $\left(A^{2^{o p}}\right)$ in a dual manner.

Definition 3.3. Let $\mathcal{C}$ be an additive category, $X$ a complex in $C h^{n-1}(\mathcal{C})$

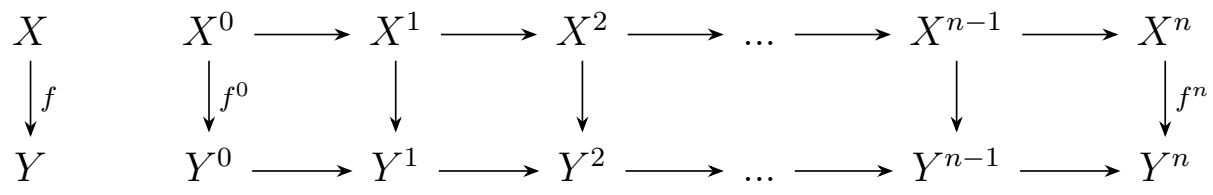

The mapping cone $C=C(f) \in C h^{n-1}(\mathcal{C})$ is

$$
X^{0} \stackrel{d_{c}^{-1}}{\longrightarrow} X^{1} \oplus Y^{0} \stackrel{d_{c}^{0}}{\longrightarrow} \ldots \stackrel{d_{c}^{n-2}}{\longrightarrow} X^{n} \oplus Y^{n-1} \stackrel{d_{c}^{n-1}}{\longrightarrow} Y^{n}
$$

where

$$
d_{c}^{k}:=\left[\begin{array}{cc}
-d_{X}^{k+1} & 0 \\
f^{k+1} & d_{Y}^{k}
\end{array}\right]: X^{k+1} \oplus Y^{k} \longrightarrow X^{k+2} \oplus Y^{k+1}
$$

for each $k \in\{-1,0,1, \ldots, n-1\}$. In particular

$$
d_{c}^{-1}=\left[\begin{array}{c}
-d_{X}^{0} \\
f^{0}
\end{array}\right] \text { and } d_{c}^{n-1}=\left[\begin{array}{ll}
f^{n} & d_{Y}^{n-1}
\end{array}\right] .
$$

(1) the diagram $f: X \longrightarrow Y$ is called $n$-pullback diagram of $Y$ along $f^{n}$ if the sequence $\left(d_{c}^{-1}, \ldots, d_{c}^{n-2}\right)$ is an $n$-kernel of $d_{C}^{n-1}$;

(2) the diagram $f: X \longrightarrow Y$ is called $n$-pushout diagram of $X$ along $f^{0}$ if the sequence $\left(d_{c}^{0}, \ldots, d_{c}^{n-1}\right)$ is an $n$-cokernel of $d_{C}^{-1}$;

(3) the diagram $f: X \longrightarrow Y$ is called $n$-bicartesian (or, $n$-exact diagram) if the sequence $C(f)=\left(d_{c}^{-1}, d_{C}^{0}, \ldots, d_{c}^{n-1}\right)$ is an $n$-exact sequence;

Proposition 3.4. Let $\mathcal{C}$ be an additive category. Suppose that we are given an n-pushout diagram

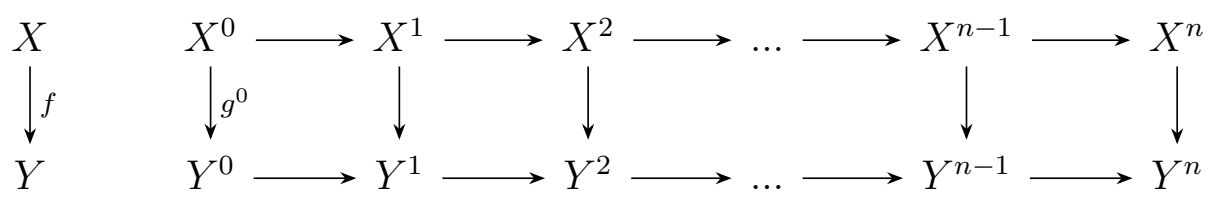


and let $k \in\{0,1, \ldots, n-1\}$. If $d_{Y}^{k+1}$ is a weak cokernel of $d_{Y}^{k}$, then $d_{X}^{k+1}$ is a weak cokernel of $d_{X}^{k}$

Proof. Put $C:=C(f)$ and let $u: X^{k+1} \longrightarrow M$ be a morphism such that $d_{X}^{k} u=0$. Consider the solid part of the following commutative diagram;

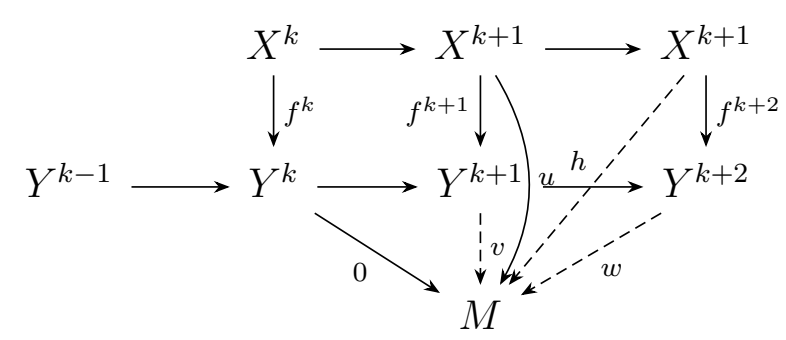

Given that $d_{C}^{k}: X^{k+1} \oplus Y^{k} \longrightarrow X^{k+2} \oplus Y^{k+1}$ is a weak cokernel of $d_{C}^{-1}: X^{k} \oplus Y^{k-1} \longrightarrow$ $X^{k+1} \oplus Y^{k}$, there exist morphisms $v: Y^{k+1} \oplus M$ and $h: X^{k+2} \longrightarrow M$ such that $d_{Y}^{k} v=0$ and $u-f^{k+1} v=d_{X}^{k+1} h$. Since $d_{Y}^{k+1}$ is a weak cokernel of $d_{Y}^{k}$ there exists a morphism $w: Y^{k+2} \longrightarrow M$ such that $v=d_{Y}^{k+1} w$. Therefore we have

$$
\begin{gathered}
\\
u=d_{X}^{k+1} h+f^{k+1} v \\
=d_{X}^{k+1} h+f^{k+1}\left(d^{k+1} w\right) \\
=d_{X}^{k+1}\left(h+f^{k+2} w\right)
\end{gathered}
$$

This shows that $d_{X}^{k+1}$ is a weak cokernel of $d_{X}^{k}$.

Proposition 3.5. Let $\mathcal{C}$ be an additive category, $g: X \longrightarrow Z$ a morphism of complexes in $C h^{n-1}(\mathcal{C})$ and suppose there exists an n-pushout diagram of $X$ along $g^{0}$

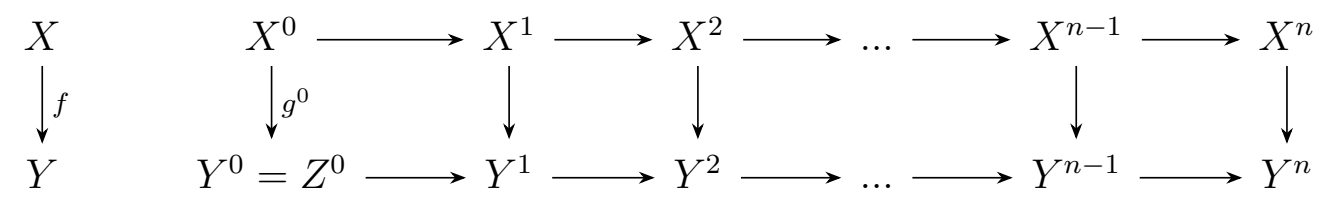

Then, there exists a morphism of complexes $p: Y \longrightarrow Z$ such that $p^{0}=1_{Z^{0}}$ and a homotopy $h: f p \longrightarrow g$ with $h^{1}=0$. Moreover, these properties determine $p$ uniquely up to homotopy.

Proof. Let $h^{1}: X^{1} \longrightarrow Z^{0}$ be the zero morphism, $p^{0}=1_{Z^{0}}$ and $C:=C(f)$. Inductively, suppose that $0 \leq k \leq n$ and that for all $l \leq k$ we have constructed a morphism $p^{l}: Y^{l} \longrightarrow Z^{l}$ such that $d_{Y}^{l-1} p^{l}=p^{l-1} d_{Z}^{l-1}$ and a morphism $h^{l+1}: X^{l+1} \longrightarrow Z^{l}$ such that $f^{l} p^{l}-g^{l}=h^{l} d_{Z}^{l-1}+d_{X}^{l} h^{l+1}$.

We claim that the composition

$$
X^{k} \oplus Y^{k-1} \stackrel{\left[\begin{array}{cc}
-d_{X}^{k} & 0 \\
f^{k} & d_{Y}^{k-1}
\end{array}\right]}{\longrightarrow} X^{k+1} \oplus Y^{\left.k \stackrel{\left[g^{k+1}-h^{k+1} d_{Z}^{k}\right.}{~} p^{k} d_{Z}^{k}\right]} Z^{k+1}
$$

vanishes. Indeed, on one hand we have

$$
f^{k}\left(p^{k} d_{Z}^{k}\right)=\left(g^{k}+d_{X}^{k} h^{k+1}\right) d_{Z}^{k}=d_{X}^{k}\left(g^{k+1}-h^{k+1} d_{Z}^{k}\right) .
$$

On the other hand, we have

$$
d_{Y}^{k-1}\left(p^{k} d_{Z}^{k}\right)=p^{k-1} d_{Z}^{k-1} d_{Z}^{k}=0
$$


The claim follows.

Next, since $d_{C}^{k}$ is a weak cokernel of $d_{C}^{k-1}$, there exists a morphism $p^{k+1}: Y^{k+1} \longrightarrow Z^{k+1}$ such that $d_{Y}^{k} p^{k+1}=p^{k} d_{Z}^{k}$ and a morphism $h^{k+2}: X^{k+2} \longrightarrow Y^{k+1}$ such that

$$
g^{k+1}+h^{k+1} d_{Z}^{k}=-d_{X}^{k+1} h^{k+2}+f^{k+1} p^{k+1} .
$$

This finishes the induction step, and the construction of the required morphism $p: Y \longrightarrow Z$. Moreover, $h: f p \longrightarrow g$ is a homotopy (note that $h^{n+1}=0$ ).

Proposition 3.6. Let $\mathcal{C}$ be an additive category and $g^{0}: X^{0} \longrightarrow Z^{0}$ a morphism in $\mathcal{C}$. Suppose that there exists an n-pushout diagram of $X \in C h^{n-1}(\mathcal{C})$ along $g^{0}$

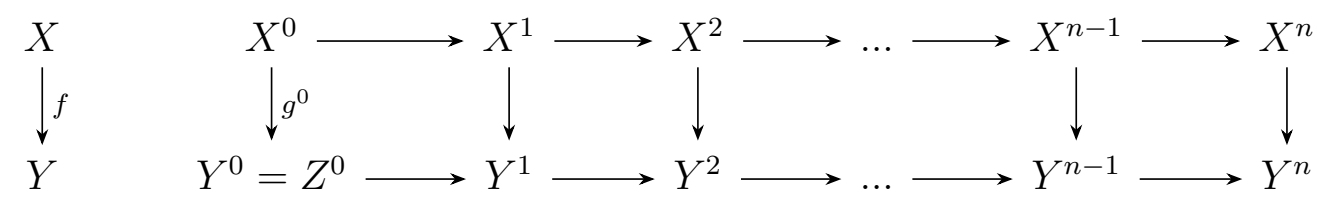

Then, the following statements hold:

(i) There exists an n-pushout diagram $\hat{f}: X \longrightarrow \hat{Y}$ of $X$ along $g^{0}$ such that for every morphism $g: X \longrightarrow Z$ of complexes lifting $g^{0}$ and such that $Z \in C h^{n-1}(\mathcal{C})$ there exists a morphism of complexes $p: Y \longrightarrow Z$ such that $p^{0}=1_{Z^{0}}$ and $\hat{f} p=g$.

(ii) For each $2 \leq k \leq n$ the morphism $\hat{f}^{k}$ is a split monomorphism.

(iii) We have $\hat{Y}=Y \oplus X^{0}$ for a contractible complex $X^{0} \in C h^{n-1}(\mathcal{C})$.

We call the morphism $\hat{f}: X \longrightarrow \hat{Y}$ a good n-pushout diagram of $X$ along $g^{0}$.

Proof. If $n=1$ the result is trivial, so we may assume that $n \geq 2$. For $C \in \mathcal{C}$ and $k \in Z$, let $i_{k}(C)$ be the complex with $d^{k}=1_{C}$ and which is 0 in each degree different from $k$ and $k+1$. We define $X^{\prime}:=\bigoplus_{k=2}^{n} i_{k-1}\left(X^{k}\right)$ and $\hat{Y}:=Y \oplus X^{0}$. Note that $\hat{Y}^{0}=Y^{0}$ and that $X^{0}$ is contractible complex. It readily follows that the diagram

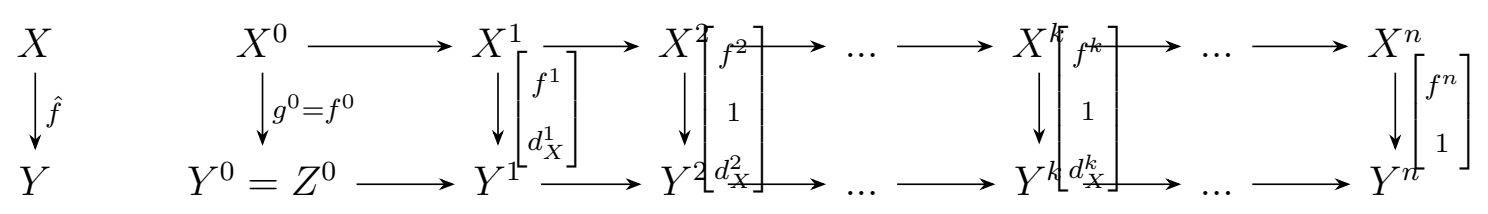

commutes. Observe that for each $2 \leq k \leq n$ the morphism $\hat{f}^{k}$ is a split monomorphism. Using Proposition 3.5, it is easy to show that $\hat{f}$ has the required factorization property; the details are left to the reader.

Proposition 3.7. Let $\mathcal{C}$ be an additive category, $g: Z \longrightarrow Y$ and $f: Y \longrightarrow X$ are morphisms in $\mathrm{Ch}^{n-1}(\mathcal{C})$. Then we have the following statements:

(i) If $f: Y \longrightarrow X$ is an n-pullback diagram of $X$ along $f^{n}$ and $g: Z \longrightarrow Y$ is an n-pullback diagram of $Y$ along $g^{n}$, then $f g: Z \longrightarrow X$ is an $n$-pullback diagram of $X$ along $f^{n} g^{n}$.

(ii) If $f: Y \longrightarrow X$ is an n-pullback diagram of $X$ along $f^{n}$ and $f g: Z \longrightarrow X$ is an $n$-pullback diagram of $X$ along $f^{n} g^{n}$, then $g: Z \longrightarrow Y$ is an n-pullback diagram of $Y$ along $g^{n}$.

Proof. We have a commutative diagram 


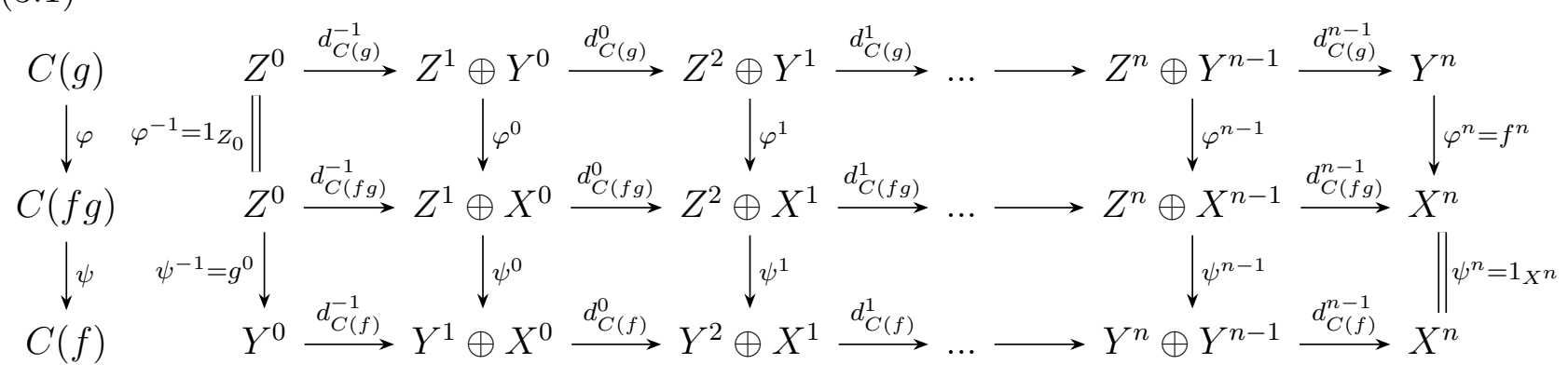

where

$$
\varphi^{k}=\left[\begin{array}{cc}
1_{Z^{k+1}} & 0 \\
0 & f^{k}
\end{array}\right], \psi^{k}=\left[\begin{array}{cc}
g^{k+1} & 0 \\
0 & 1_{X^{k}}
\end{array}\right]
$$

for $k \in\{1,2, \ldots, n-1\}$.

(i) $f: Y \longrightarrow X$ is an $n$-pullback diagram of $X$ along $f^{n}$ and $g: Z \longrightarrow Y$ is an $n$-pullback diagram of $Y$ along $g^{n}$, then $\left(d_{C(f)}^{-1}, \ldots, d_{C(f)}^{n-2}\right)$ and $\left(d_{C(g)}^{-1}, \ldots, d_{C(g)}^{n-2}\right)$ are $n$-kernels of $d_{C(f)}^{n-1}$ and $d_{C(g)}^{n-1}$ respectively, $d_{C(f)}^{-1}$ and $d_{C(g)}^{-1}$ are monomorphisms. Hence $d_{C(f g)}^{-1}$ is a monomorphism. Indeed, let $u: M \longrightarrow Z^{0}$ be a morphism such that $d_{C(f g)}^{-1} u=0$. Then $d_{Z}^{0} u=0$. But $0=\psi^{0} d_{C(f g)}^{-1} u=$ $d_{C(f)}^{-1} g^{0} u$, this implies $g^{0} u=0$ since $d_{C(f)}^{-1}$ is a monomorphism. Thus $d_{C(g)}^{-1} u=0$ so is $u$ since $d_{C(g)}^{-1}$ is a monomorphism. So, $d_{C(f g)}^{-1}$ is a monomorphism. Thus $d_{C(f g)}^{i-1}$ is a weak kernel of $d_{C(f g)}^{i}$ for $i=\{0, \ldots, n-1\}$ (we consider $X^{-1}, Y^{-1}, Y^{-1}, X^{n+1}, Y^{n+1}, Y^{n+1}$ as 0 objects). Indeed, let

$$
\left[\begin{array}{c}
u^{i+1} \\
v^{i}
\end{array}\right]: M \longrightarrow Z^{i+1} \oplus X^{i}
$$

be a morphism such that $d_{C(f g)}^{i}\left[\begin{array}{c}u^{i+1} \\ v^{i}\end{array}\right]=0$, hence we have $d_{C(f)}^{i} \psi^{i}\left[\begin{array}{c}u^{i+1} \\ v^{i}\end{array}\right]=0$. Then

$$
d_{Z}^{i+1} u^{i+1}=0, f^{i+1} g^{i+1} u^{i+1}+d_{X}^{i} v^{i}=0,
$$

and there exists a morphism

$$
\left[\begin{array}{c}
w^{i} \\
t^{i-1}
\end{array}\right]: M \longrightarrow Y^{i} \oplus X^{i-1}
$$

such that

$$
d_{C(f)}^{i-1}\left[\begin{array}{c}
w^{i} \\
t^{i-1}
\end{array}\right]=\psi_{i}\left[\begin{array}{c}
u^{i+1} \\
v^{i}
\end{array}\right]
$$

since $d_{C(f)}^{i-1}$ is a weak kernel of $d_{C}^{i}(f)$. Then

$$
g^{i+1} u^{i+1}+d_{Y}^{i} w^{i}=0, f^{i} w^{i}+d_{X}^{i-1} t^{i-1}=v^{i} .
$$

Then, we have

$$
d_{C(g)}^{i}\left[\begin{array}{c}
u^{i+1} \\
w^{i}
\end{array}\right]=\left[\begin{array}{cc}
-d_{Z}^{i+1} & 0 \\
g^{i+1} d_{Y}^{i} &
\end{array}\right]\left[\begin{array}{c}
u^{i+1} \\
w^{i}
\end{array}\right]=\left[\begin{array}{c}
-d_{Z}^{i+1} u^{i+1} \\
g^{i+1} u^{i+1}+d_{Y}^{i} w^{i}
\end{array}\right]=0
$$

Therefore, since $d_{C(g)}^{i-1}$ is a weak kernel of $d_{C(g)}^{i}$, there exists a morhpism

$$
\left[\begin{array}{c}
s^{i} \\
h^{i-1}
\end{array}\right]: M \longrightarrow Z^{i} \oplus Y^{i-1}
$$


such that $d_{C(g)}^{i-1}\left[\begin{array}{c}s^{i} \\ h^{i-1}\end{array}\right]=\left[\begin{array}{c}u^{i+1} \\ w^{i}\end{array}\right]$. We have that

$$
u^{i+1}+d_{Z}^{i} s^{i}=0, g^{i} s^{i}+d_{Y}^{i-1} h^{i-1}=w^{i}
$$

Set

$$
\left[\begin{array}{c}
s^{i} \\
f^{i-1} h^{i-1}+t^{i-1}
\end{array}\right]: M \longrightarrow Z^{i} \oplus X^{i-1}
$$

we have

$$
d_{C(f g)}^{i-1}\left[\begin{array}{c}
s^{i} \\
f^{i-1} h^{i-1}+t^{i-1}
\end{array}\right]=\left[\begin{array}{cc}
-d_{Z}^{i} & 0 \\
f^{i} g^{i} & d_{X}^{i-1}
\end{array}\right]\left[\begin{array}{c}
s^{i} \\
f^{i-1} h^{i-1}+t^{i-1}
\end{array}\right]=\left[\begin{array}{c}
u^{i+1} \\
v^{i}
\end{array}\right] .
$$

This proves that $d_{C(f g)}^{i-1}$ is a weak kernel of $d_{C(f g)}^{i}$ for $i=\{0, \ldots, n-1\}$, thus $f g: Z \longrightarrow X$ is an $n$-pullback diagram of $Y$ along $f^{n} g^{n}$.

(ii) Because $f: Y \longrightarrow X$ is an $n$-pullback diagram of $X$ along $f^{n}$ and $f g: Z \longrightarrow X$ is an $n$-pullback diagram of $Z$ along $f^{n} g^{n},\left(d_{C(f)}^{-1}, \ldots, d_{C(f)}^{n-2}\right)$ and $\left(d_{C(f g)}^{-1}, \ldots, d_{C(f g}^{n-2}\right)$ are $n$-kernels of $d_{C(f)}^{n-1}$ and $d_{C(f g)}^{n-1}$ respectively, $d_{C(f)}^{-1}$ and $d_{C(f g)}^{-1}$ are monomorphisms, so is $d_{C(g)}^{-1}$. Hence $d_{C(g)}^{i-1}$ is a weak kernel of $d_{C(g)}^{i}$ for $i=\{0, \ldots, n-1\}$. Indeed, let

$$
\left[\begin{array}{c}
u^{i+1} \\
v^{i}
\end{array}\right]: M \longrightarrow Z^{i+1} \oplus Y^{i}
$$

be a morphism such that $d_{C(g)}^{i}\left[\begin{array}{c}u^{i+1} \\ v^{i}\end{array}\right]=0$, we have

$$
d_{Z}^{i+1} u^{i+1}=0, g^{i+1} u^{i+1}+d_{Y}^{i} v^{i}=0, d_{C(f g)}^{i} \varphi^{i}\left[\begin{array}{c}
u^{i+1} \\
v^{i}
\end{array}\right]=0
$$

Therefore, since $d_{C(f g)}^{i-1}$ is a weak kernel of $d_{C(f g)}^{i}$, there exists a morhpism

$$
\left[\begin{array}{c}
s^{i} \\
h^{i-1}
\end{array}\right]: M \longrightarrow Z^{i} \oplus X^{i-1}
$$

such that $d_{C(f g)}^{i-1}\left[\begin{array}{c}s^{i} \\ h^{i-1}\end{array}\right]=\varphi^{i}\left[\begin{array}{c}u^{i+1} \\ v^{i}\end{array}\right]$. Thus we have

$$
d_{Z}^{i} s^{i}+u^{i+1}=0, f^{i} g^{i} s^{i}+d_{X}^{i-1} h^{i-1}=f^{i} v^{i}
$$

and

$$
d_{C(f)}^{i-1}\left[\begin{array}{c}
v^{i}-g^{i} s^{i} \\
-h^{i-1}
\end{array}\right]=\left[\begin{array}{c}
d_{Y}^{i} v^{i}+d_{Y}^{i} g^{i} s^{i} \\
f^{i} v^{i}-f^{i} g^{i} s^{i}-d_{X}^{i-1} h^{i-1}
\end{array}\right]=0
$$

Therefore, since $d_{C(f)}^{i-2}$ is a weak kernel of $d_{C(f)}^{i-1}$, there exists a morhpism Therefore, since $d_{C(f g)}^{i-1}$ is a weak kernel of $d_{C(f g)}^{i}$, there exists a morhpism

$$
\left[\begin{array}{c}
w^{i-1} \\
t^{i-2}
\end{array}\right]: M \longrightarrow Y^{i-1} \oplus X^{i-2}
$$


such that $d_{C(f)}^{i-2}\left[\begin{array}{c}w^{i-1} \\ t^{i-2}\end{array}\right]=\left[\begin{array}{c}v^{i}-g^{i} s^{i} \\ -h^{i-1}\end{array}\right]$. Thus, we have $-d_{Y}^{i-1} w^{i-1}=v^{i}-g^{i} s^{i}$. Then,

$$
d_{C(g)}^{i-1}\left[\begin{array}{c}
s^{i} \\
-w^{i-1}
\end{array}\right]=\left[\begin{array}{c}
-d_{Z}^{i} s^{i} \\
g^{i} s^{i}-d_{Y}^{i-1} w^{i-1}
\end{array}\right]=\left[\begin{array}{c}
u^{i+1} \\
v^{i}
\end{array}\right]
$$

This proves that $d_{C(g)}^{i-1}$ is a weak kernel of $d_{C(g)}^{i}$ for $i=\{0, \ldots, n-1\}$, thus $g: Z \longrightarrow Y$ is an $n$-pullback diagram of $Z$ along $g^{n}$.

Proposition 3.8. Let $\mathcal{C}$ be an n-abelian category, $g: Z \longrightarrow Y$ and $f: Y \longrightarrow X$ are morphisms in $C h^{n-1}(\mathcal{C})$. If $f: Y \longrightarrow X$ is an n-pullback diagram of $X$ along $f^{n}$ and $g: Z \longrightarrow Y$ is an $n$-pullback diagram of $Y$ along $g^{n}$, then $g: Z \longrightarrow Y$ and $f: Y \longrightarrow X$ are $n$-exact diagrams if and only if $f g: Z \longrightarrow X$ is a n-exact diagram.

Proof.

$(\Rightarrow)$ This is obvious by the dual of Proposition 3.7.

$(\Leftarrow)$ Because $f: Y \longrightarrow X$ is a $n$-pullback diagram of $X$ along $f^{n},\left(d_{C(f)}^{n-2}, \ldots, d_{C(f)}^{-1}\right)$ is a $n$-kernel of $d_{C(f)}^{n-1}$ in the bottom row of Diagram (3.1). The middle row of Diagram (3.1) is an $n$-exact sequence by the definition of $n$-exact diagram and $d_{C(f)}^{n-1}$ is an epimorphism since $d_{C(f g)}^{n-1}$ is an epimorphism. By axiom $\left(A^{2^{o p}}\right)$ in Definition 3.1, the bottom row of Diagram (3.1) is an $n$ exact sequence, thus $g: Z \longrightarrow Y$ is an $n$-exact diagram. It is enough to show that $d_{C(g)}^{n-1}$ is an epimorphism by axiom $\left(A^{2^{o p}}\right)$ in Definition 3.1 since $\left(d_{C(g)}^{-1}, \ldots, d_{C(g)}^{n-2}\right)$ is an $n$-kernel of $d_{C(g)}^{n-1}$. Indeed, let $u: Y^{n} \longrightarrow M$ be a morphism such that

$$
u d_{C(g)}^{n-1}=\left[\begin{array}{ll}
u g^{n} & u d_{Y}^{n-1}
\end{array}\right]=0 .
$$

Then,

$$
\left[\begin{array}{ll}
u & 0
\end{array}\right]: Y^{n} \oplus X^{n-1} \longrightarrow M
$$

is a morphism such that $\left[\begin{array}{ll}u & 0\end{array}\right] d_{C(f)}^{n-2}=0$. Therefore, since $d_{C(f)}^{n-1}$ is the cokernel of $d_{C(f)}^{n-2}=0$, there exists a morphism $h: X^{n} \longrightarrow M$ such that $h\left(f^{n} d_{X}^{n-1}\right)=\left[\begin{array}{ll}u & 0\end{array}\right]$. Hence $h=0$ since

$$
h d_{C(f)}^{n-1}=\left[\begin{array}{ll}
h d_{X}^{n-1} & h f^{n} g^{n}
\end{array}\right]=0
$$

and $d_{C(f g)}^{n-1}$ is an epimorphism. Thus, $u=0$. This proves our assertion.

Proposition 3.9. Let $\mathcal{C}$ be an $n$-abelian category and

$$
X: X^{0} \stackrel{f^{0}}{\longrightarrow} X^{1} \stackrel{f^{1}}{\longrightarrow} X^{0} \stackrel{f^{0}}{\longrightarrow} \ldots X^{0} \stackrel{f^{n-1}}{\longrightarrow} X^{n} \stackrel{f^{n}}{\longrightarrow} X^{n+1}
$$

a complex in $\mathcal{C}$. Then, for every $k \in\{0,1, \ldots, n\}$ and every $l \in\{1,2, \ldots, n\}$ there exist morphisms $g_{k}^{l}: Y_{k}^{l} \longrightarrow Y_{k}^{l-1}$ (with $Y_{k}^{0}=X^{k}$ ) and $p_{k}^{l-1}: Y_{k}^{l-1} \longrightarrow Y_{k+1}^{l}$ satisfying the following properties:

(i) For every $k \in\{0,1, \ldots, n\}$ the diagram

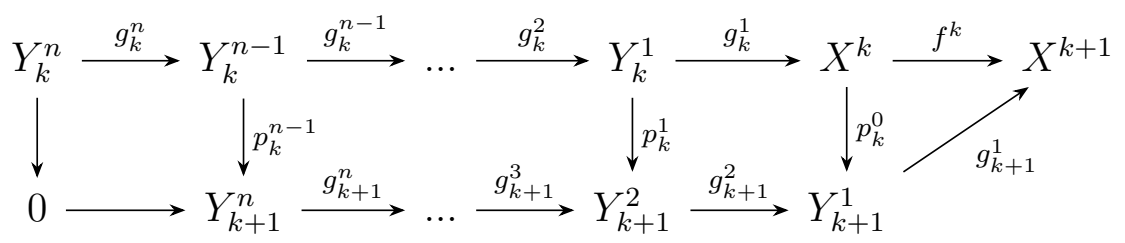

commutes.

(ii) The sequence $\left(g_{k}^{n}, \ldots, g_{k}^{1}\right)$ is an n-kernel of $f^{k}$. 
(iii) The diagram

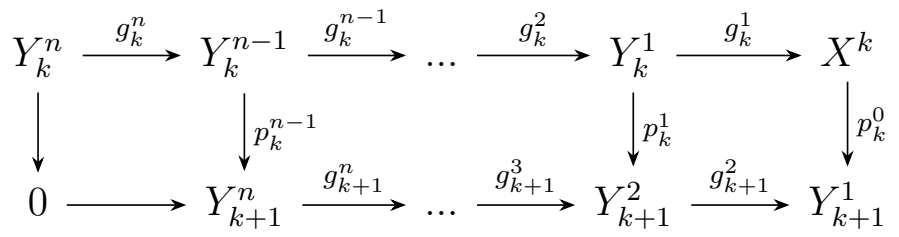

is an $n$-pullback diagram.

Moreover the morphism

$$
\left[\begin{array}{ll}
p_{k}^{0} & g_{k+1}^{2}
\end{array}\right]: X^{k} \oplus Y_{k+1}^{2} \longrightarrow Y_{k+1}^{1}
$$

is an epimorphism for every $k$ if and only if the complex $X$ is a right $n$-exact sequence. In this case we can choose the objects $Y^{l} k, 1 \leq l \leq n$ and morphisms $g_{k}^{l}, 1 \leq l \leq n$ in such a way that the Diagram (3.2) is both n-pullback and n-pushout diagram.

(iv) If the complex $X$ is a right n-exact sequence and $k \neq 0$, then the sequence $\left(g_{k}^{k-1}, \ldots, g_{k}^{1}, f_{k}, \ldots, f^{n}\right)$ is an $n$-cokernel of the morphism $g_{k}^{k}$.

Proof. Let

$$
X: X^{0} \stackrel{f^{0}}{\longrightarrow} X^{1} \stackrel{f^{1}}{\longrightarrow} X^{0} \stackrel{f^{0}}{\longrightarrow} \ldots X^{0} \stackrel{f^{n-1}}{\longrightarrow} X^{n} \stackrel{f^{n}}{\longrightarrow} X^{n+1}
$$

be a right $n$-exact sequence. Since $f^{n}$ is an epimorphism, there exists an $n$-exact sequence

$$
Y_{n}^{n} \stackrel{g_{n}^{n}}{\longrightarrow} Y_{n}^{n-1} \stackrel{g_{n}^{n-1}}{\longrightarrow} \ldots \stackrel{g_{n}^{2}}{\longrightarrow} Y_{n}^{1} \stackrel{g_{n}^{1}}{\longrightarrow} X^{n} \stackrel{f^{n}}{\longrightarrow} X^{n+1}
$$

This implies that the diagram

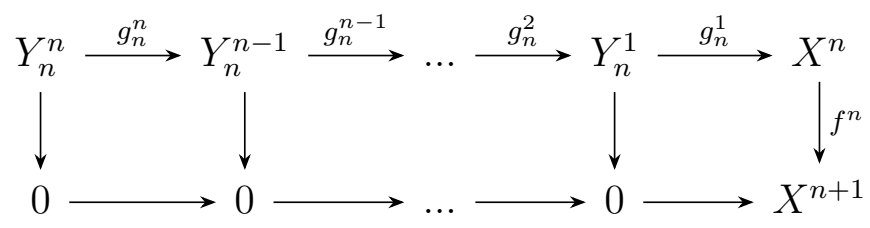

is both an $n$-pullback diagram and an $n$-pushout diagram. Now by induction assume that for $1 \leq k \leq n$ we have the following commutative diagram with the required properties.

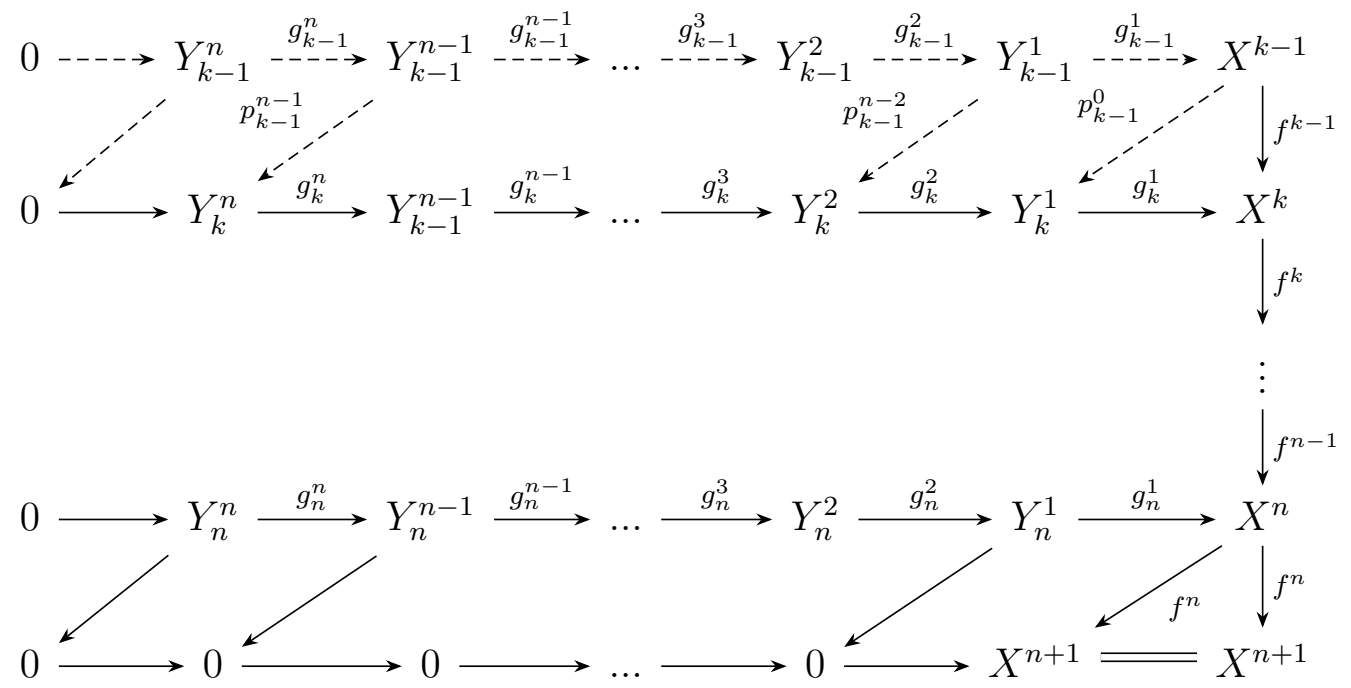


Since $f^{k} f^{k-1}=0$ and $g_{k}^{1}$ is a weak kernel of $f^{k}$, there exists a morphism $p_{k-1}^{0}: X^{k-1} \longrightarrow Y_{k}^{1}$ such that $f^{k-1}=g_{k}^{1} p_{k-1}^{0}$. Note that the dotted morphisms in the diagram are obtained by taking n-pullback of the complex

$$
0 \longrightarrow Y_{k}^{n} \stackrel{g_{k}^{n}}{\longrightarrow} Y_{k}^{n-1} \stackrel{g_{k}^{n-1}}{\longrightarrow} \ldots \stackrel{g_{k}^{3}}{\longrightarrow} Y_{k}^{2} \stackrel{g_{k}^{2}}{\longrightarrow} Y_{k}^{1}
$$

along $p_{k-1}^{0}$. It is easy to see that for every $1 \leq k \leq n$ the induced morphism

$$
\left[\begin{array}{ll}
p_{k-1}^{0} & g_{k+1}^{2}
\end{array}\right]: X^{k-1} \oplus Y_{k}^{2} \longrightarrow Y_{k}^{1}
$$

in the above diagram is an epimorphism and hence the diagram is both an $n$-pullback diagram and an $n$-pushout diagram. Now all required properties are followed from basic properties of $n$-pullback and $n$-pushout. Now assume that for every $k$

$$
\left[\begin{array}{ll}
p_{k}^{0} & g_{k+1}^{2}
\end{array}\right]: X^{k} \oplus Y_{k+1}^{2} \longrightarrow Y_{k+1}^{1}
$$

is an epimorphism. We show that the complex $X$ is a right $n$-exact sequence. It is clear that $f^{n}$ is an epimorphism. Let $u: X^{k+1} \longrightarrow M$ be a morphism such that $u f^{k}=0$. Since

$$
\left[\begin{array}{ll}
p_{k}^{0} & g_{k+1}^{2}
\end{array}\right]: X^{k} \oplus Y_{k+1}^{2} \longrightarrow Y_{k+1}^{1}
$$

is an epimorphism, $u g_{k+1}^{1}=0$. By assumption

$$
0 \longrightarrow\left(Y_{k+2}^{1}, M\right) \longrightarrow\left(X^{k+1} \oplus Y_{k+2}^{2}, M\right) \longrightarrow\left(y_{k+1}^{1} \oplus Y_{k+2}^{3}, M\right)
$$

is exact and so there is a morphism $v: Y^{1} \oplus k+2 \longrightarrow M$ such that $u=v p_{k+1}^{0}$ and $v g_{k+2}^{2}=0$. Since $g_{k+2}^{1}$ is a weak cokernel of $g_{k+2}^{2}$ there is a morphism $w: X^{k+2} \longrightarrow M$ such that $v=w g_{k+2}^{1}$. Now it is easy to see that $u=w f^{k+1}$ and hence $f^{k+1}$ is a weak cokernel of $f^{k}$. Then the complex $X$ is a right $n$-exact sequence.

\section{N-ABELIAN CATEGORY}

Remark 4.1. Let $\mathcal{C}$ be an $n$-abelian category. An immediate consequence of axioms $\left(A^{1}\right)$ and $\left(A^{2}\right)$ (resp. $\left(A^{2^{o p}}\right)$ ) is that every monomorphism (resp. epimorphism) in $\mathcal{C}$ appears as the leftmost (resp. rightmost) morphism in some $n$-exact sequence.

Note that 1-abelian categories are precisely abelian categories in the usual sense. It is easy to see that abelian categories are idempotent complete; thus, if $n=1$, then axiom $\left(A^{0}\right)$ in Definition 3.1 is redundant. However, if $n \geq 2$, then axiom $\left(A^{0}\right)$ is independent from the remaining axioms as shown by the following example.

Example 4.2. Let $n \geq 2$ and $K$ be a field. Consider the full subcategory $\nu$ of mod $K$ given by the finite dimensional $K$-vector spaces of dimension different from 1 . Then, $\nu$ is not idempotent complete but it satisfies axioms $\left(A^{1}\right),\left(A^{2}\right)$ and $\left(A^{2^{o p}}\right)$.

Proof. Firstly, note that $\nu$ is an additive subcategory of $\bmod K$. The fact that $\nu$ is not idempotent complete is obvious (consider the idempotent $0 \oplus 1_{K}: K^{2} \longrightarrow K^{2}$ whose kernel is one-dimensional, for example). Let us show that $\nu$ satisfies axiom $\left(A^{1}\right)$. Indeed, let $f: V \longrightarrow W$ be a morphism in $\nu$. If cokernel $f$ has dimension different from 1 , then

$$
V \longrightarrow W \longrightarrow \operatorname{Cokerf} \longrightarrow 0 \longrightarrow \ldots \longrightarrow 0
$$


gives an $n$-cokernel of $f$ in $\nu$. If cokernel $f$ has dimension 1 , then we can construct an $n$-cokernel of $f$ in $\nu$ by a commutative diagram

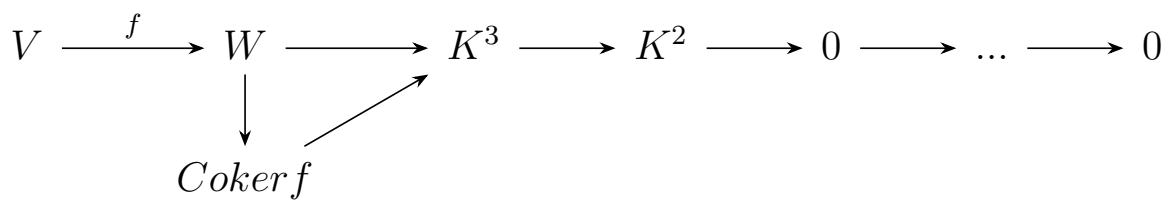

where Coker $f \longrightarrow K^{3} \longrightarrow K^{2}$ is a kernel-cokernel pair. We can construct an $n$-kernel of $f$ in a dual manner. This shows that $\nu$ satisfies axiom $\left(A^{1}\right)$. That $\nu$ satisfies axioms $\left(A^{2}\right)$ and $\left(A^{2^{o p}}\right)$ follows from Proposition 2.10 since contractible complexes with $n+2$ terms are in particular n-exact sequences by Proposition 2.9.

Lemma 4.3. Let $\mathcal{C}$ be an idempotent complete additive category and suppose that we are given a sequence of morphisms in $\mathcal{C}$ of the form

$$
A \stackrel{f}{\longrightarrow} B \stackrel{g}{\longrightarrow} C \stackrel{h}{\longrightarrow} D
$$

If $g$ is a weak cokernel of $f$, and $h$ is both a split epimorphism and a cokernel of $g$, then $f$ admits a cokernel in $\mathcal{C}$.

Proof. Since $h$ is a split epimorphism there exists a morphism $i: D \longrightarrow C$ such that $i h=1_{D}$. It follows that the morphism $e:=1_{C}-h i$ is idempotent. Since the category $\mathcal{C}$ is idempotent complete, there exists an object $E \in \mathcal{C}$ and morphisms $r: C \longrightarrow E$ and $s: E \longrightarrow C$ such that $s r=1_{E}$ and $r s=e$. Note that this implies that $\mathrm{r}$ is an epimorphism and $s h=0$ for we have

$$
r(s h)=(1-h i) h=h-h=0 .
$$

We claim that $g \mathrm{r}$ is a cokernel of $f$. Indeed, let $u: B \longrightarrow B^{\prime}$ be a morphism such that $f u=0$. Since $g$ is a weak cokernel of $f$ there exists a morphism $v: C \longrightarrow B^{\prime}$ such that $u=g v$. It follows that

$$
u=g v=g(1-h i) v=(g r)(s v) .
$$

This shows that $g r$ is a weak cokernel of $f$. It remains to show that $g r$ is an epimorphism. For this, let $w: E \longrightarrow E^{\prime}$ be a morphism such that $(g r) w=0$. Since $h$ is a cokernel of $g$ there exists a morphism $x: D \longrightarrow E^{\prime}$ such that $r w=h x$. It follows that

$$
w=(s r) w=s(h x)=0 .
$$

This shows that $g r$ is an epimorphism. Therefore $g r$ is a cokernel of $f$.

Proposition 4.4. Let $\mathcal{M}$ be an additive category which satisfies axioms $\left(A^{0}\right)$ and $\left(A^{1}\right)$ in Definition 3.1, and let $X$ a complex in $C h^{n-1}(\mathcal{C})$. If for all $1 \leq k \leq n-1$ the morphism $d^{k}$ is a weak cokernel of $d^{k-1}$, then $d^{n-1}$ admits a cokernel in $\mathcal{M}$.

Proof. If $n=1$, then the result follows trivially from axiom $\left(A^{1}\right)$ in Definition 3.1. Hence we may assume that $n \geq 2$. By axiom $\left(A^{1}\right)$ in Definition 3.1 there exists an $n$-cokernel 


$$
\left(d^{k}: X^{k} \longrightarrow X^{k+1} \mid n \leq k \leq 2 n-1\right)
$$

of $d^{n-1}$. Using axiom $\left(A^{1}\right)$ in Definition 3.1 again together with the factorization property of weak cokernels, we obtain a commutative diagram

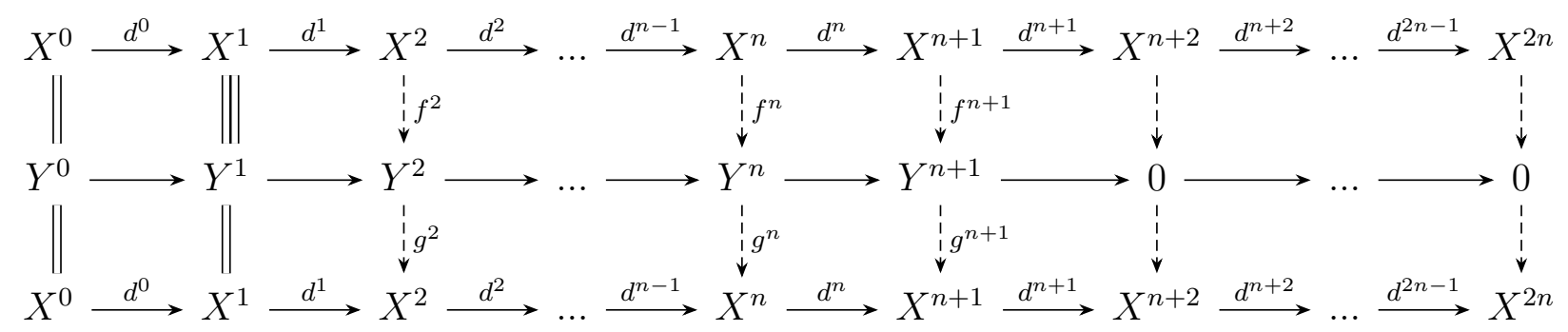

in which the middle row gives an $n$-cokernel of $d^{0}$. The Comparison Lemma 2.8 implies that there exists a morphism $h: X^{2 n} \longrightarrow X^{2 n-1}$ such that $h d^{2 n-1}=1$. Hence we may apply Lemma 4.3 and reduce the length of the $n$-cokernel of $d^{n-1}$ by one morphism. Proceeding inductively, we deduce that $d^{n-1}$ has a cokernel in $\mathcal{M}$.

The importance of axiom $\left(A^{0}\right)$ in Definition 3.1 becomes apparent in the following result, which asserts that $n$-abelian categories have $n$-pushout diagrams and $n$-pullback diagrams.

Theorem 4.5. (Existence of n-pushout diagrams). Let $\mathcal{M}$ be an additive category which satisfies axioms $\left(A^{0}\right)$ and $\left(A^{1}\right)$ in Definition 3.1. Let $X$ a complex in $C h^{n-1}(\mathcal{C})$, and a morphism $f: X^{0} \longrightarrow Y^{0}$. Then, the following statements hold:

(i) Then, there exists an n-pushout diagram

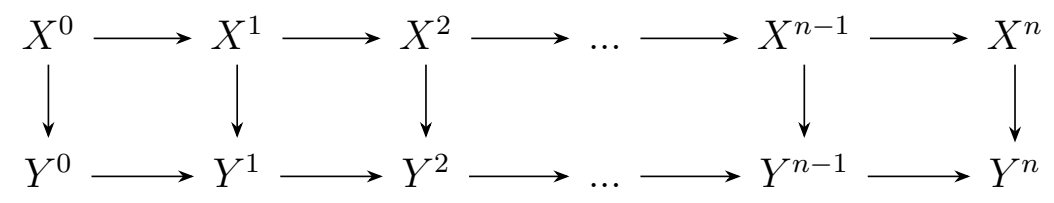

(ii) Suppose, moreover, that $\mathcal{M}$ is an n-abelian category. If $d_{X}^{0}$ is a monomorphism, then $d_{Y}^{0}$ is a monomorphism.

Proof.

(i) We shall construct the complex $Y$ inductively. Set $f^{0}:=f$ and

$$
d_{c}^{-1}=\left[\begin{array}{c}
-d_{X}^{0} \\
f^{0}
\end{array}\right]: X^{0} \longrightarrow X^{1} \oplus Y^{0}
$$

Let $0 \leq k \leq n-2$ and suppose that for each $l \leq k$ we have constructed an object $Y^{l}$ and morphisms $f^{l}: X^{l} \longrightarrow Y^{l}$ and $d_{Y}^{l-1}: Y^{l-1} \longrightarrow Y^{l}$ such that $d_{C}^{l-2} d_{C}^{l-1}=0$ where

$$
d_{c}^{l-1}:=\left[\begin{array}{cc}
-d_{X}^{l} & 0 \\
f^{l} & d_{Y}^{l-1}
\end{array}\right]: X^{l} \oplus Y^{l-1} \longrightarrow X^{l+1} \oplus Y^{l}
$$

(compare with Definition 2.5). Then, by axiom $\left(A^{1}\right)$ in Definition 3.1, the morphism $d_{C}^{k-1}$ has a weak cokernel

$$
g^{k}:=\left[\begin{array}{ll}
f^{k+1} & d_{Y}^{k}
\end{array}\right]: X^{k+1} \oplus Y^{k} \longrightarrow X^{k+2} \oplus Y^{k+1} .
$$


We claim that

$$
d_{c}^{k}:=\left[\begin{array}{cc}
-d_{X}^{k+1} & 0 \\
f^{k+1} & d_{Y}^{k}
\end{array}\right]: X^{k+1} \oplus Y^{k} \longrightarrow X^{k+2} \oplus Y^{k+1}
$$

is also a weak cokernel of $d_{C}^{k-1}$. Indeed, it is readily verified that $d_{C}^{k-1} d_{C}^{k}=0$. Let $u$ : $X^{k+1} \oplus Y^{k} \longrightarrow M$ be a morphism such that $d_{C}^{k-1} u=0$. Since $g^{k}$ is a weak cokernel of $d_{C}^{k-1}$ , there exists a morphism $v: Y^{k+1} \longrightarrow M$ such that $u=g^{k} v$. It follows that the following diagram is commutative:

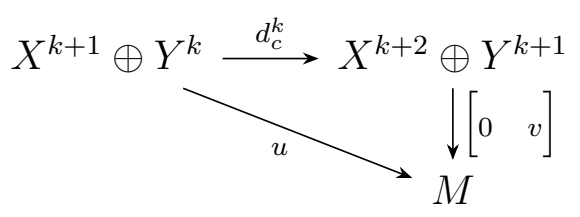

This shows that $d_{C}^{k}$ is a weak cokernel of $d_{C}^{k-1}$. Finally, Proposition 4.4 implies that the morphism $d_{C}^{n-2}$ admits a cokernel $d_{C}^{n-1}: X^{n} \oplus Y^{n-1} \longrightarrow Y^{n}$.

This shows that the tuple $\left(d_{C}^{0}, d_{C}^{1}, \ldots, d_{C}^{n-1}\right)$ is a weak cokernel of $d_{C}^{-1}$. The existence of the required commutative diagram follows from the fact that $C$ is a complex.

(ii) Finally, suppose that $\mathcal{M}$ is $n$-abelian and $d_{X}^{0}$ is a monomorphism. Note that this implies that $d_{C}^{-1}$ is also a monomorphism. Then, axiom $\left(A^{2}\right)$ in Definition 3.1 implies that the $C$ is an $n$-exact sequence. In order to show that $d_{Y}^{0}$ is a monomorphism, let $u: M \longrightarrow Y^{0}$ be a morphism such that $u d_{Y}^{0}=0$. It follows that the composition

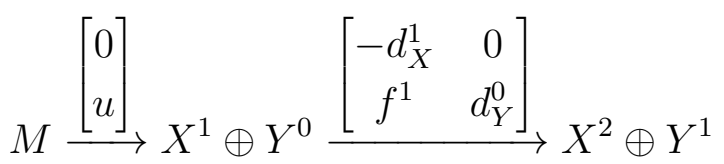

vanishes. Given that $d_{C}^{-1}$ is a kernel of $d_{C}^{0}$, there exists a morphism $v: M \longrightarrow X^{0}$ such that $v d_{X}^{0}=0$ and $v f^{0}=u$. Since $d_{X}^{0}$ is a monomorphism, we have $u=0$. This shows that $d_{Y}^{0}$ is a monomorphism.

Theorem 4.6. Let $\mathcal{C}$ be an additive category and $n$ a positive integer. Then, the $n$-abelian categories in which every n-exact sequence is contractible are precisely the semisimple categories.

Proof. Suppose that $\mathcal{C}$ is a semisimple category. We only show that $\mathcal{C}$ is idempotent complete. It is straightforward to verify that $\mathcal{C}$ satisfies the remaining axioms of $n$-abelian categories, the fact that every $n$-exact sequence in $\mathcal{C}$ is contractible follows immediately from Proposition 2.10. Let us show then that $\mathcal{C}$ is idempotent complete. Let $e: A \longrightarrow A$ be an idempotent in $\mathcal{C}$. Since $\mathcal{C}$ is semisimple, $e$ factors as $e=p i$ where $p: A \longrightarrow B$ is a split epimorphism and $i: B \longrightarrow A$ is a split monomorphism.

We claim that $i p=1_{B}$. Indeed, let $h: A \longrightarrow B$ be a morphism such that $i h=1_{B}$. Given that $e^{2}=e$ we have

$$
p=p(i h)=e h=e^{2} h=(p i p i) h=p(i p)
$$

Since $p$ is an epimorphism we have $i p=1_{B}$ as claimed. This shows that $\mathcal{C}$ is idempotent complete. 
Conversely, suppose that $\mathcal{C}$ is an $n$-abelian category in which every $n$-exact sequence is contractible and let $f: A \longrightarrow B$ be a morphism in $\mathcal{C}$. We claim that $f$ admits both a kernel and a cokernel in $\mathcal{C}$. Indeed, by axiom $\left(A^{1}\right)$ in Definition 3.1 there exists an $n$-cokernel $\left(f^{1}, \ldots f^{n}\right)$ of $f$. By hypothesis, the epimorphism $f^{n}$ must split. Then, Lemma 4.3 implies that $f^{n-2}$ has a cokernel in $\mathcal{C}$. By applying this argument inductively we deduce that $f$ admits a cokernel in $\mathcal{C}$. By duality, $f$ also admits a kernel in $\mathcal{C}$. The remaining part of the proof is classical, compare for example with the proof of Proposition 2.12.

We need to show that $f$ factors as $f=p i$ where $p$ is a split epimorphism and $i$ is a split monomorphism. Given that $f$ has both a kernel and a cokernel in $\mathcal{C}$, is easy to construct a commutative diagram

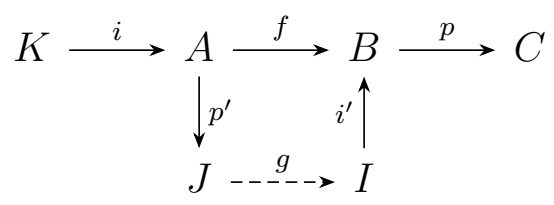

where $i$ is a kernel of $f$, and $p$ is a cokernel of $f$, and the sequences $K \longrightarrow A \longrightarrow J$ and $I \longrightarrow B \longrightarrow C$ are kernel-cokernel pairs. We claim that $g$ is an isomorphism, for which it is enough to show that is both a monomorphism and an epimorphism as all such morphisms split by hypothesis. By duality we only need to show that $g$ is an epimorphism.

Let $h: I \longrightarrow I^{\prime}$ be a morphism such that $g h=0$. Firstly, by Theorem 4.5 there exists a commutative diagram

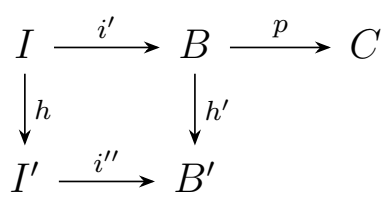

where $i^{\prime \prime}$ is a monomorphism. Secondly, we claim that $f h^{\prime}=0$. Indeed, we have

$$
f h^{\prime}=\left(p^{\prime} g i^{\prime}\right) h^{\prime}=p^{\prime}(g h) i^{\prime \prime}=0
$$

Therefore, since $p$ is a cokernel of $f$, there exists a morphism $j: C \longrightarrow B^{\prime}$ such that $p j=h^{\prime}$. It follows that

$$
h i^{\prime \prime}=i^{\prime} h^{\prime}=i^{\prime}(p j)=0
$$

Finally, since $i^{\prime \prime}$ is a monomorphism, we have $h=0$. This shows that $g$ is an epimorphism.

Corollary 4.7. Let $m$ and $n$ be two distinct positive integers and $\mathcal{C}$ an additive category. If $\mathcal{C}$ is both $m$-abelian and $n$-abelian, then $\mathcal{C}$ is a semisimple category.

Proof. Without loss of generality we may assume that $m<n$. By Theorem 4.6 and Proposition 2.10 it is enough to show that every monomorphism in $\mathcal{C}$ splits. Let $f^{0}: X^{0} \longrightarrow X^{1}$ be a monomorphism in $\mathcal{C}$ and let $\left(f^{1}, \ldots, f^{n}\right)$ be an $n$-cokernel of $f^{0}$, and $\left(g^{1}, \ldots, g^{m}\right)$ be an $m$-cokernel of $f^{0}$. It follows that there exists a commutative diagram 


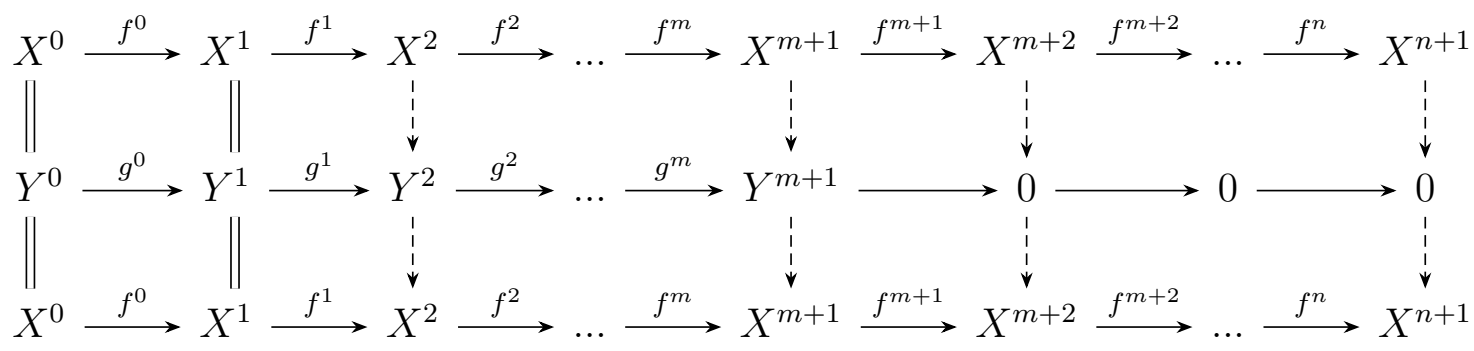

By the Lemma 2.8 there exists a morphism $h: X^{n+1} \longrightarrow X^{n}$ such that $h f^{n}=1$. Thus $f^{n}$ is a split epimorphism. Then, as $\left(f^{1}, \ldots, f^{n}\right)$ is an $n$-exact sequence by axiom $\left(A^{2}\right)$ in Definition 3.1, the dual of Proposition 2.10 implies that $f^{0}$ is a split monomorphism.

Proposition 4.8. Let $\mathcal{C}$ be an additive category which satisfies axioms $\left(A^{0}\right)$ and $\left(A^{1}\right)$ in Definition 3.1, let $X$ be a complex in $C h^{n-1}(\mathcal{C})$ such that the morphism $d_{X}^{k}$ is a weak cokernel of $d_{X}^{k-1}$ for $1 \leq k \leq n-1$. Let $f^{0}: X^{0} \longrightarrow Y^{0}$ be a morphism. Then there exists a cokernel $d_{X}^{n}: X^{n} \longrightarrow X^{n+1}$ of $d_{X}^{n-1}$ such that $\left(d_{X}^{1}, \ldots, d_{X}^{n}\right)$ is an $n$-cokernel of $d_{X}^{0}$, and for any $n$-pushout diagram

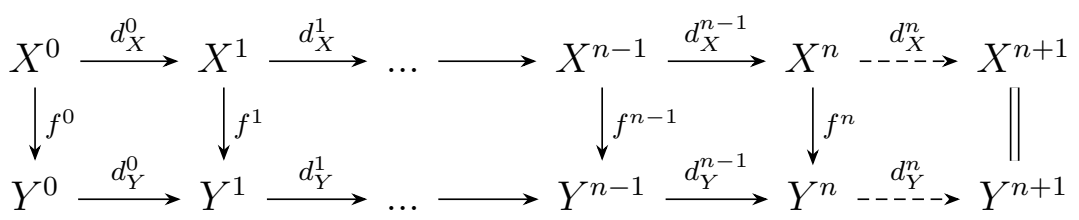

of $\left(d_{X}^{0}, \ldots, d_{X}^{n-1}\right)$ along $f^{0}$, there exists a cokernel $d_{Y}^{n}: Y^{n} \longrightarrow X^{n+1}$ of $d_{Y}^{n-1}$ such that $\left(d_{Y}^{1}, \ldots, d_{X}^{n}\right)$ is an n-cokernel of $d_{Y}^{0}$ and $d_{X}^{n}=d_{Y}^{n} f^{n}$. Moreover, if $d_{X}^{0}$ is a monomorphism, both rows are $n$-exact sequences.

Proof. The existence of $d_{X}^{n}: X^{n} \longrightarrow X^{n+1}$ of $d_{X}^{n-1}$ is immediately by Lemma 4.3. Since $d_{C}^{n-1}$ is a cokernel of $d_{C}^{n-2}$, there exists an unique morphism $d_{Y}^{n}: Y^{n} \longrightarrow X^{n+1}$ such that $d_{X}^{n}=d_{Y}^{n} f^{n}$ and $d_{Y}^{n} d_{Y}^{n-1}=0$. Since $d_{X}^{n}$ is an epimorphism so is $d_{Y}^{n}$. It remains to show that $d_{Y}^{n}$ is a cokernel of $d_{Y}^{n-1}$. Let $u: Y^{n} \longrightarrow M$ be a morphism such that $u d_{Y}^{n-1}=0$. Then

$$
\left(u f^{n}\right) d_{X}^{n-1}=u\left(d_{Y}^{n-1} f^{n-1}\right)=0 .
$$

Since $d_{X}^{n}$ is a cokernel of $d_{X}^{n-1}$, there exists a morphism $v: X^{n+1} \longrightarrow M$ such that $u f^{n}=v d_{X}^{n}$. It follows that

$$
u f^{n}=v d_{X}^{n}=\left(v d_{Y}^{n}\right) f^{n}, u d_{Y}^{n-1}=0=\left(v d_{Y}^{n}\right) d_{Y}^{n-1}
$$

Since $d_{C}^{n-1}$ is a cokernel of $d_{C}^{n-2}, u=v d_{Y}^{n}$. This shows that the epimorphism $d_{Y}^{n}$ is a cokernel of $d_{Y}^{n-1}$.

We show that the morphism $d_{Y}^{k+1}$ is a weak cokernel of $d_{Y}^{k}$ for $2 \leq k \leq n$, this shows that $\left(d_{Y}^{1}, \ldots, d_{Y}^{n}\right)$ is an $n$-cokernel of $d_{Y}^{0}$. Indeed, let $u: Y^{k+1} \longrightarrow M$ be a morphism such that $u d_{Y}^{k}=$ 0 . Then $u f^{k+1} d_{X}^{k}=u d_{Y}^{k} f^{k}=0$, there exists $v^{k+2}: X^{k+2} \longrightarrow M$ such that $u f^{k+1}=v^{k+2} d_{X}^{k+1}$ since $d_{X}^{k+1}$ is a weak cokernel of $d_{X}^{k}$. Then there exists morphisms $v^{k+3}: X^{k+2} \longrightarrow M$ and 
$u^{k+2}: Y^{k+2} \longrightarrow M$ (we set $X^{n+1}=0$ ) such that $u^{k+2} d_{Y}^{k+1}=u$ since $d_{C}^{k+1}$ is a weak cokernel of $d_{C}^{k}$. This shows that $d_{Y}^{k+1}$ is a weak cokernel of $d_{Y}^{k}$.

Moreover, if $d_{X}^{0}$ is a monomorphism, by Theorem $(i i) 4.5, n$-pushout preserve monorphism, then $d_{Y}^{0}$ is a monomorphism. By axiom $\left(A^{2}\right)$ in Definition 3.1, both the two rows are $n$-exact sequences.

\section{REFERENCES}

[1] M. Auslander and S.O. Smal $\phi$, Almost split sequences in subcategories. J. Algebra. 69(2) (1981) 426-454. https://doi.org/10.1016/0021-8693(81)90214-3.

[2] M.P. Brodmann and R.Y. Sharp, Local cohomology: an algebraic introduction with geometric applications, Cambridge Studies in Advanced Mathematics, vol. 60, Cambridge University Press, Cambridge, 2012.

[3] T. Bühler, Exact categories. Expo. Math. 28(1) (2010) 1-69. https://doi.org/10.1016/j.exmath. 2009. 04.004.

[4] R. Ebrahimi, A. Nasr-Isfahani, Representation of n-abelian categories in abelian categories, J. Algebra. 563 (2020) 352-375. https://doi.org/10.1016/j.jalgebra.2020.07.010.

[5] P. Fredy, Abelian categories: An introduction to the theory of functors. Harper's Series in Modern Mathematics. Harper and Row Publishers, New York, 1994.

[6] L. Frerick and D. Sieg, Exact categories in functional analysis, Preprint (2010). http://www.math. uni-trier.de/abteilung/analysis/HomAlg.pdf.

[7] A. Grothendieck, Sur quelques points D'algèbre homologique. Tohoku Math. J. 9(2) (1957), 119-183.

[8] G. Jasso, n-Abelian and n-exact categories, Math. Z. 283 (2016) 703-759. https://doi.org/10.1007/ s00209-016-1619-8.

[9] A. Neeman, The derived category of an exact category. J. Algebra. 135(2) (1990), 388-394. https://doi. org/10.1016/0021-8693(90)90296-z.

[10] L. Ribes, P. Zalesskii, Profinite Groups, in: Profinite Groups, Springer Berlin Heidelberg, Berlin, Heidelberg, 2000: pp. 19-77. https://doi.org/10.1007/978-3-662-04097-3_2.

[11] J.J. Rotman, An introduction to homological algebra. Springer, New York, 2009.

[12] C.A. Weibel, An introduction to homological algebra, Cambridge Studies in Advanced Mathematics. Cambridge University Press, Cambridge, 2011.

[13] Q. Zheng, J. Wei, Quotient categories of n-Abelian categories, Glasgow Math. J. 62 (2020) 673-705. https://doi.org/10.1017/S0017089519000417.

[14] Z. Panyue and B. Zhu, n-Abelian quotient categories. J. Algebra. 527 (2019), 264-279. https://doi.org/ $10.1017 / \mathrm{s} 0017089519000417$. 